$10,11,05$

\title{
Исследование термодинамических свойств и фазовых переходов В трехвершинной сильноразбавленной антиферромагнитной модели Поттса методом Монте-Карло
}

\author{
(C) А.К. Муртазаев ${ }^{1,2}$, А.Б. Бабаев ${ }^{1,3}$, Г.Я. Атаева ${ }^{1,4, q}$ \\ ${ }^{1}$ Институт фризики им. Х.И. Амирханова Даг НЦ РАН, \\ Махачкала, Россия \\ 2 Дагестанский государственный университет, \\ Махачкала, Россия \\ ${ }^{3}$ Дагестанский государственный педагогический университет, \\ Махачкала, Россия \\ ${ }^{4}$ Дагестанский государственный университет народного хозяйства, \\ Махачкала, Россия \\ ฯ E-mail: ataeva20102014@mail.ru
}

(Поступила в Редакцию 11 мая 2016 г.)

\begin{abstract}
Методом Монте-Карло исследованы термодинамические свойства и фазовые переходы в двумерной сильноразбавленной антиферромагнитной модели Поттса на треугольной решетке. Рассмотрены системы с линейными размерами $L \times L=N, L=18-48$. С использованием метода кумулянтов Биндера четвертого порядка показано, что внесение немагнитных примесей в спиновую систему, описываемую двумерной антиферромагнитной моделью Поттса, приводит к смене фазового перехода первого на фазовый переход второго рода.
\end{abstract}

Исследование выполнено при финансовой поддержке РФФИ в рамках научного проекта № 16-02-00214-а и №16-32-00105-мол-а.

DOI: 10.21883/FTT.2017.01.43963.175

Изучение фазовых переходов (ФП) и связанных с ними критических явлений (КЯ) традиционно привлекают к себе весьма активное внимание физиков. Неидеальные черты присущие реальным системам оказывают значительное влияние на фазовые переходы и критические явления в магнитных системах, в частности присутствие немагнитных примесей в системе может изменить род фазового перехода, и эта проблема остается актуальной в течение последних двадцати лет $[1,2]$. Теоретические и лабораторные методы сталкиваются с рядом проблем при попытке рассмотрения данного вопроса, и, в связи с этим, данная проблема исследуется на основе моделей, которые отображают основные свойства реального тела и позволяют провести численное или аналитическое описание системы и таким образом описать фазовый переход. В итоге с развитием вычислительной физики и с применением методов Монте-Карло стало возможно изучать более реалистичные модели и учитывать усложняющие факторы всегда присутствующие в реальных материалах [2,3]. В качестве таких моделей реальных физических систем, могут выступать, двумерные статистические системы, такие как модель Поттса, критическое поведение которой весьма богато и интересно само по себе. Наиболее интересным на сегодня объектом для изучения является антиферромагнитная модель Поттса, которая мало изучена, о ней нет достоверных данных о влиянии немагнитных примесей на ФП, неустановлен класс универсальности критического поведения, нет сведений о зависимости критических индексов от концентрации немагнитных примесей, особенно когда беспорядок реализован в виде вмороженных немагнитных примесей [4]. Достоверным фактом является лишь то, что в чистой беспримесной системе наблюдается фазовый переход первого рода [5].

В данной работе исследуются фазовые переходы в двумерной сильноразбавленной антиферромагнитной модели Поттса на треугольной решетке при концентрации спинов $p=0.70$ и 0.65. При этом рассматривались системы с периодическими граничными условиями и линейными размерами $L \times L=N, L=18-48$. Модель Поттса в чистом состоянии $(p=1.00)$ и в слаборазбавленном режиме $(p=0.90,0.80)$ была исследована в работе [6].

Для вывода системы в равновесное состояние вычислялось время релаксации $\tau_{0}$ для всех рассмотренных систем. Для моделирования спиновой конфигурации был применен стандартный алгоритм Метрополиса [7] в сочетании с однокластерным алгоритмом Вольфа метода Монте-Карло [8].

При построении сильно неупорядоченной модели Поттса следует учесть следующие особенности: в узлах треугольной решетки расположены спины $S_{i}$, которые могут находиться в одном из $q>2$ состояний, и немагнитные примеси; немагнитные примеси расположены случайно и фиксированы в узлах решетки. 
Гамильтониан подобной системы имеет вид [5].

$$
H=-\frac{1}{2} J \sum_{i, j} \rho_{i} \rho_{j} \cos \theta_{i, j}, \quad S_{i}=1,2,3,
$$

где $J$ - параметр обменного антиферромагнитного взаимодействия ближайших соседей $(J<0) ; \rho_{i}=1$, если узел $i$ занят магнитным атомом, $\rho_{i}=0$, если в узле находится немагнитная примесь; $\theta_{i, j}-$ угол между взаимодействующими спинами $S_{i}-S_{j}$.

Для определения критических температур и рода фазового перехода был задействован метод кумулянтов Биндера четвертого порядка [9],

$$
\begin{gathered}
V_{L}(T, p)=1-\frac{\left\langle E^{4}\right\rangle_{L}}{3\left\langle E^{2}\right\rangle_{L}^{2}}, \\
U_{L}(T, p)=1-\frac{\left\langle m^{4}(T, p ; L)\right\rangle_{L}}{3\left\langle m^{2}(T, p ; L)\right\rangle_{L}^{2}},
\end{gathered}
$$

где $E$ - энергия и $m-$ намагниченность системы с линейным размером $L$. Выражения (2) и (3) позволяют определить $T_{c}(p)$ с большой точностью в фазовых переходах первого и второго рода соответственно. Данный метод хорошо зарекомендовал себя и при определении рода ФП. Известным фактом является несколько отличительных черт характерных для ФП [10]: для ФП первого рода характерно то, что усредненная величина $V_{L}(T, p)$ стремится к некоторому нетривиальному значению $V^{*}$ согласно выражению

$$
V(T, p)=V^{*}+b L^{-d},
$$

при $L \rightarrow \infty$ и $T=T_{c}(L)$, где $V^{*}$ отлична от $2 / 3$; минимальная величина $U_{L, \min }\left(T=T_{\min }, p\right)$ расходится $U_{L, \min }\left(T=T_{\min }, p\right) \rightarrow-\infty$ при $L \rightarrow \infty$; при ФП второго рода усредненная величина $V_{L}(T, p)$ при $T=T_{c}(L)$ будет стремиться к значению $2 / 3$, а кривые температурной зависимости кумулянтов Биндера $U_{L}(T, p)$ будут иметь четко выраженную точку пересечения. Методика определения рода ФП этим методом подробно описана в работах [11-15].

Характерные зависимости кумулянтов Биндера $U_{L}(T, p)$ для сильноразбавленной антиферромагнитной модели Поттса от температуры для систем с разными линейными размерами $L$ приведены на рис. 1. Здесь и далее на всех рисунках погрешность данных не превосходит размеров символов используемых для обозначения зависимости. Как видно из рис. 1 в критической области наблюдается четко выраженная точка пересечения, что и свидетельствует о ФП второго рода. Кроме того, этот рисунок демонстрирует насколько точно можно определить критическую температуру $T_{c}$. На рис. 2 показаны зависимости кумулянтов Биндера $V_{L}(T, p)$ от температуры для систем с разными

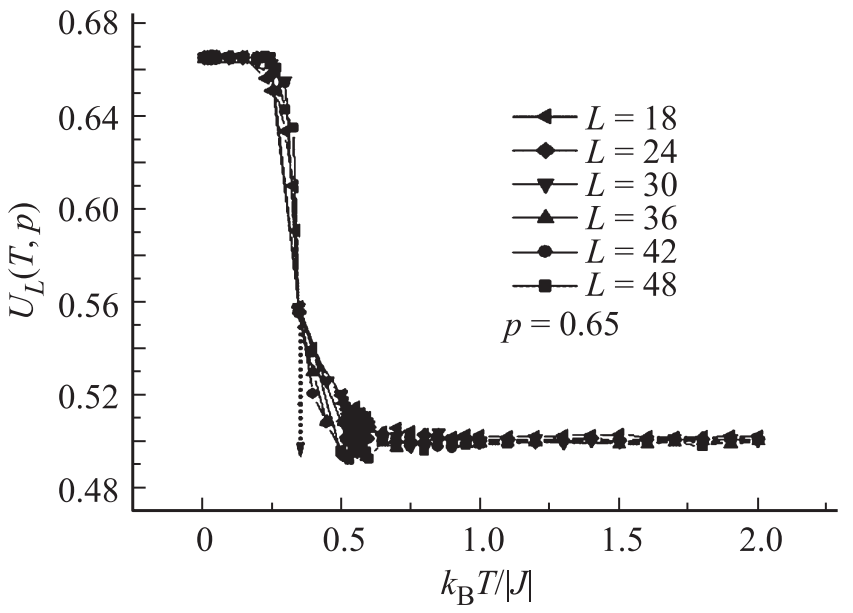

Рис. 1. Температурная зависимость кумулянтов Биндера $U_{L}(T, p)$ разбавленной АФ-модели Поттса при $p=0.65$.

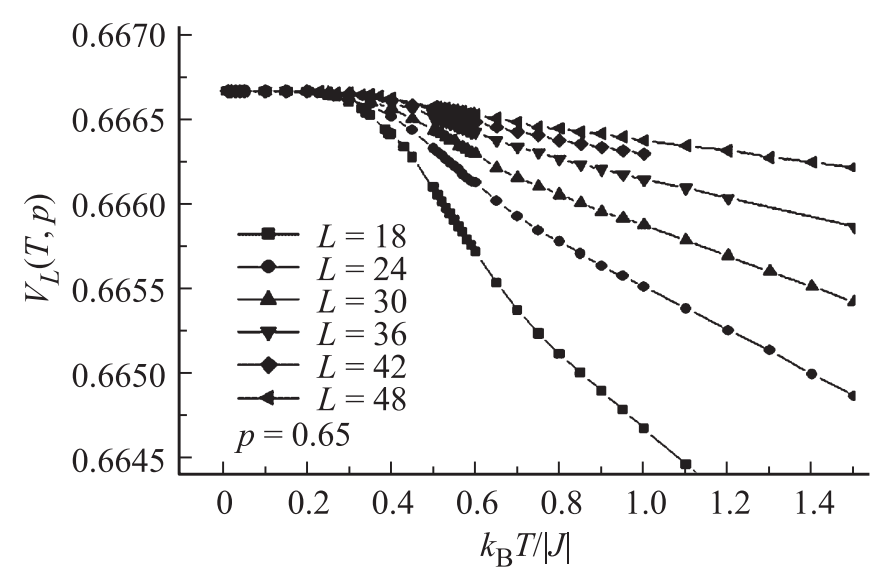

Рис. 2. Температурная зависимость кумулянтов Биндера $V_{L}(T, p)$ разбавленной АФ-модели Поттса при $p=0.65$.

линейными размерами при $p=0.65$. Из рис. 2 хорошо видно стремление величины $V_{L}(T, p)$ к $2 / 3$ при $L \rightarrow \infty$.

Такое поведение, как отмечалось выше, характерно для ФП второго рода [10]. Аналогичное поведение наблюдалось и для систем при концентрации спинов $p=0.70$. Определенные методом кумулянтов Биндера критические температуры $T_{N}(p)$ в единицах $|J| / k_{\mathrm{B}}$ равны: $T_{N}(1.00)=0.940(1), T_{N}(0.90)=0.79(1), T_{N}(0.80)$ $=0.65(2), T_{N}(0.70)=0.42(3), T_{N}(0.65)=0.35(4)$.

Для наблюдения за температурным ходом поведения теплоемкости и восприимчивости нами использовались флуктуационные соотношения [16]

$$
\begin{gathered}
C=\left(N K^{2}\right)\left(\left\langle U^{2}\right\rangle-\langle U\rangle^{2}\right), \\
\chi=(N K)\left(\left\langle m^{2}\right\rangle-\langle m\rangle^{2}\right),
\end{gathered}
$$

где $K=|J| / k_{\mathrm{B}} T, N=L^{2}$ - число магнитных узлов, $U-$ внутренняя энергия, $m$ - параметр порядка системы, угловые скобки означают термодинамическое усреднение. В качестве параметра порядка антиферромагнитной 


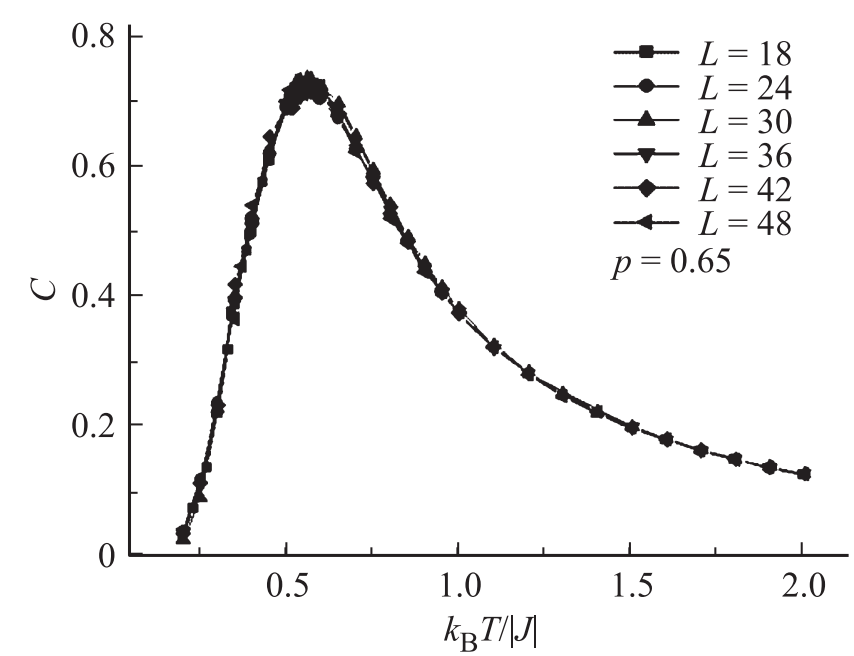

Рис. 3. Температурная зависимость теплоемкости $C$ разбавленной АФ-модели Поттса при $p=0.65$.

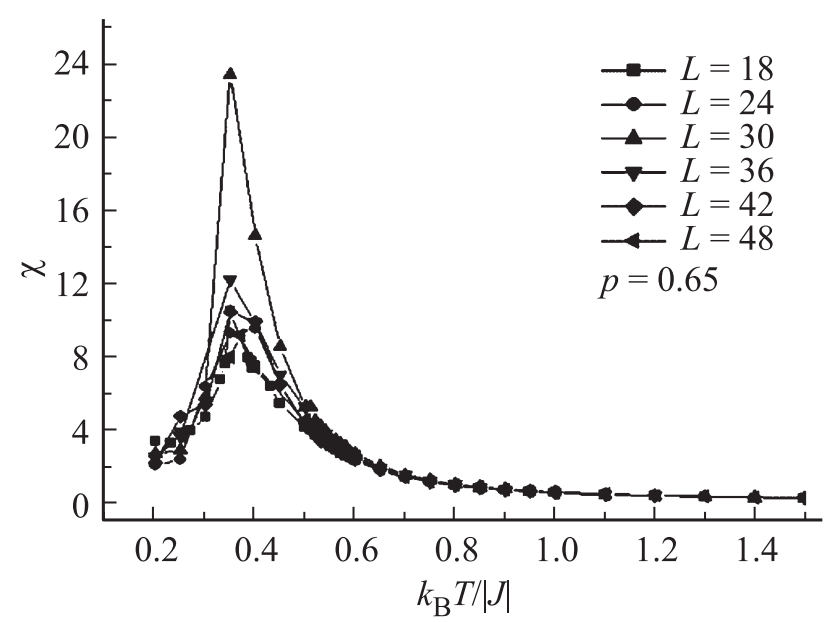

Рис. 4. Температурная зависимость восприимчивости $\chi$ разбавленной АФ-модели Поттса при $p=0.65$.

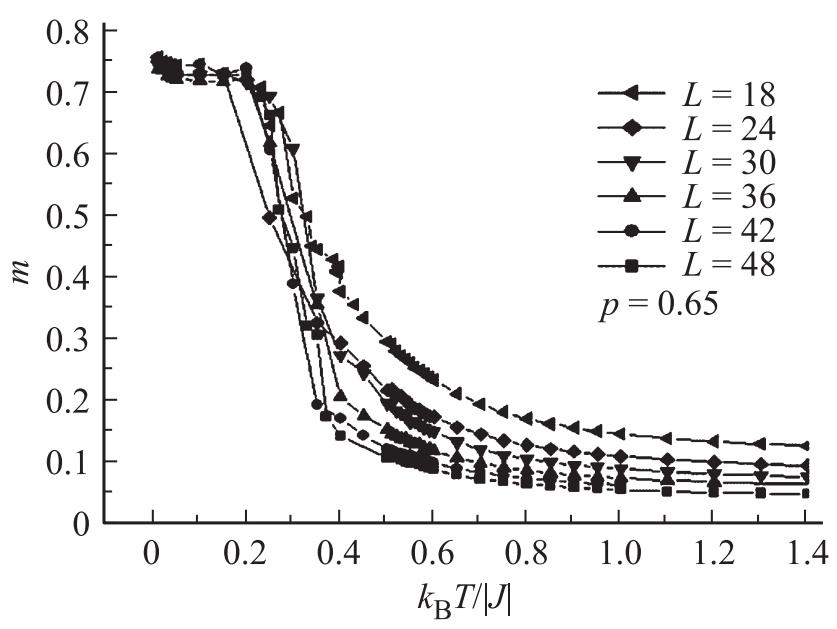

Рис. 5. Температурная зависимость намагниченности $m$ разбавленной АФ-модели Поттса при $p=0.65$.

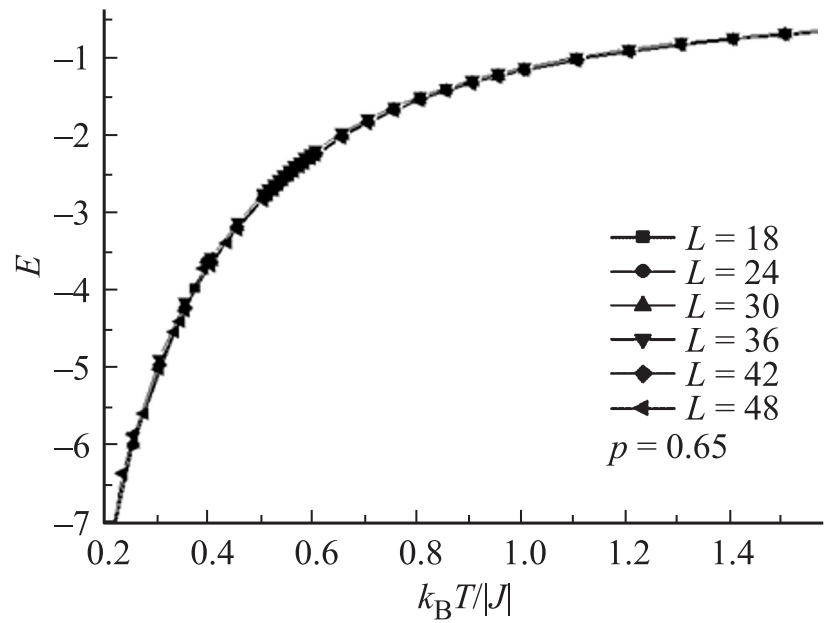

Рис. 6. Температурная зависимость энергии $E$ разбавленной АФ-модели Поттса при $p=0.65$.

$\left(m_{\mathrm{AF}}\right)$ модели Поттса использовалось следующее выражение [17]:

$$
m_{\mathrm{AF}}=\left\langle\frac{3}{2} \sum_{\alpha \neq \beta \neq \gamma}\left(\frac{\left(N_{\alpha}+N_{\beta}+N_{\gamma}\right.}{N}-\frac{1}{3}\right)^{2}\right\rangle^{1 / 2},
$$

где $N_{\alpha}=\left\{N_{1}, N_{2}, N_{3}\right\}, N_{1}$ - число спинов в состоянии с $q=1, N_{2}$ - число спинов в состоянии с $q=2, N_{3}$ число спинов в состоянии с $q=3, N_{\alpha}, N_{\beta}, N_{\gamma}$ - число спинов в подрешетке $A, B$ и $C$ соответственно, $N=L^{2}$.

На рис. 3, 4 и 5 представлены характерные зависимости теплоемкости $C$, восприимчивости $\chi$ и намагниченности $m$ от температуры $T$ для систем с разными линейными размерами.

Отметим, что в зависимостях теплоемкости $C$ и восприимчивости $\chi$ от температуры для всех исследуемых нами систем проявляются четко выраженные максимумы, и эти максимумы в пределах погрешности приходят на одну температуру. Так же нами была исследована зависимость энергии $E$ от температуры для двумерной сильноразбавленной антиферромагнитной модели Поттса на треугольной решетке при $p=0.65$, которая показана на рис. 6. Отсутствие гистерезиса в ходе кривых энергетической зависимости говорит о том, что данное поведение характерно для фазового перехода второго рода.

Таким образом, в настоящей работе с соблюдением единой методики исследовано влияние сильного беспорядка на фазовые переходы в двумерной антиферромагнитной модели Поттса на треугольной решетке при $p=0.70$ и 0.65 Полученные данные свидетельствуют о том, что внесение сильного вмороженного беспорядка в виде немагнитных примесей в двумерную чистую структуру, описываемой антиферромагнитной моделью Поттса, приводит к смене ФП первого рода на ФП второго рода. Такая смена рода фазового перехода связана с тем, что примеси приводят к подавлению флуктуационной неустойчивости. 


\section{Список литературы}

[1] В.С. Доценко. УФН 165, 5, 481 (1995).

[2] Р. Фольк, Ю. Головач, Т. Яворский. УФН 173, 175 (2003).

[3] А.К. Муртазаев, И.К. Камилов, А.Б. Бабаев. ЖЭТФ 126, 1377 (2004).

[4] X. Qian, Y. Deng, W. Blote. Phys. Rev. E 72, 056132 (2005).

[5] F.Y. Wu. Rev. Mod. Phys. 54, 235 (1982).

[6] А.К. Муртазаев, А.Б. Бабаев, Г.Я. Атаева. ФТТ 57, 7, 1410 (2015).

[7] N. Metropolis, W. Rosenbluth. J. Chem. Phys. 21, 6, 1087 (1953).

[8] U. Wolff. Phys. Lett. 62, 361 (1989).

[9] K. Eichhorn, K. Binder. J. Phys.: Cond. Matter 8, 5209 (1996).

[10] D. Loison, K.D. Schotte. Eur. Phys. J. 5, 735 (1998).

[11] A.K. Murtazaev, A.B. Babaev, G.Y. Aznaurova. Solid State Phenomena 168-169, 357 (2011).

[12] A.K. Murtazaev, A.B. Babaev. J. Magn. Magn. Mater. 324, 3870 (2012).

[13] А.К. Муртазаев, А.Б. Бабаев, Г.Я. Азнаурова. ФТТ 50, 703, (2008).

[14] A.K. Murtazaev, A.B. Babaev. J. Magn. Magn. Mater. 321, 2630 (2009).

[15] А.К. Муртазаев, А.Б. Бабаев. Письма в ЖЭТФ 99, 618 (2014).

[16] P. Peczac, A.M. Ferrenberg, D.P. Landau. Phys.Rev. B 43, 6087 (1991).

[17] Y. Saito. J. Phys. A 15, 1885 (1982). 